Open Access

\title{
Endorsement of International Consensus Radiochemistry Nomenclature Guidelines
}

Philip Elsinga ${ }^{1 *}$, Angelika Bischof Delaloye ${ }^{2}$, Arturo Chiti ${ }^{3,4}$, Stefaan Vandenberghe ${ }^{5}$, Francesco Giammarile ${ }^{6}$ and Ignasi Carrio ${ }^{7}$

\author{
* Correspondence: \\ p.h.elsinga@umcg.nl \\ This article is co-published in the \\ European Journal of Nuclear \\ Medicine and Molecular Imaging, \\ EJNMMI Research, EJNMMI Physics, \\ EJNMMI Radiopharmacy and \\ Chemistry, and European Journal of \\ Hybrid Imaging, and is available at \\ https://doi.org/10.1186/s41181-018- \\ 0050-3, https://doi.org/10.1007/ \\ s00259-018-4243-5, https://doi.org/ \\ 10.1186/s13550-018-0469-2, https:// \\ doi.org/10.1186/s40658-018-0237-4 \\ and https://doi.org/10.1186/s41824 \\ 018-0048-9 respectively. \\ ${ }^{1}$ Department of Nuclear Medicine \\ and Molecular Imaging, University \\ Medical Center Groningen, \\ University of Groningen, Hanzeplein \\ 1, Groningen 9713, EZ, The \\ Netherlands \\ Full list of author information is \\ available at the end of the article
}

With the open letter published in EJNMMI Radiopharmacy and Chemistry by an international working group of experts, the EJNMMI journal family endorses the application of the guidelines in contributions to the journals.

Attached to the letter by the working group is a 3-page summary highlighting the most relevant issues used in the notation of radiopharmaceuticals and related terms.

The full paper with all recommendations is published in "Consensus nomenclature rules for radiopharmaceutical chemistry - setting the record straight, Coenen, H.H., Gee A.D. et al., Nuclear Medicine and Biology, Volume 55, v - xi (2017) (https:// doi.org/10.1016/j.nucmedbio.2017.09.004)."

All Editors-in Chiefs of the EJNMMI journals strongly recommend all manuscripts meet these guidelines upon submission. In addition, all reviewers will be asked to be aware of the guidelines and wherever possible to check if the guidelines are followed.

Editors-in-Chief,

Philip Elsinga

Angelika Bischof Delaloye

Arturo Chiti

Stefaan Vandenberghe

Francesco Giammarile

Ignasi Carrio

Authors' contributions

All authors read and approve the final manuscript

Competing interests

The authors declare that they have no competing interests.

\section{Publisher's Note}

Springer Nature remains neutral with regard to jurisdictional claims in published maps and institutional affiliations.

\section{Author details}

'Department of Nuclear Medicine and Molecular Imaging, University Medical Center Groningen, University of Groningen, Hanzeplein 1, Groningen 9713, EZ, The Netherlands. ${ }^{2}$ Nuclear Medicine Service Centre Hospitalier Universitaire Vaudois, Bugnon 46, Lausanne 1011, Switzerland. ${ }^{3}$ Department of Biomedical Sciences, Humanitas University, Milan, Italy. ${ }^{4}$ Department of Diagnostic Imaging, Humanitas Clinical and Research Center - IRCCS, Milan, Italy. ${ }^{5}$ MEDISIP-ELIS-IBITECH, Ghent University, Corneel Heymanslaan 10, Ghent 9000, Belgium. ${ }^{6}$ Nuclear Medicine and Diagnostic Imaging Section, Division of Human Health, International Atomic Energy Agency, Vienna, Austria.

${ }^{7}$ Department of Radiology and Medical Physics, Hospital de Sant Pau, Barcelona, Spain.

Received: 8 November 2018 Accepted: 8 November 2018

Published online: 16 April 2019

(๑) The Author(s). 2019 Open Access This article is distributed under the terms of the Creative Commons Attribution 4.0 International License (http://creativecommons.org/licenses/by/4.0/), which permits unrestricted use, distribution, and reproduction in any medium provided you give appropriate credit to the original author(s) and the source, provide a link to the Creative Commons license, and indicate if changes were made. 